\title{
Pursuit of Optimal Targeted Temperature Management for Patients With Out-of-Hospital Cardiac Arrest
}

\author{
Tetsuya Matoba, MD, PhD; Hiroyuki Tsutsui, MD, PhD
}

$\mathbf{R}$ esuscitation from out-of-hospital cardiac arrest $(\mathrm{OHCA})$ is one of the remaining challenges in emergency cardiovascular medicine. Approximately 1 million people in Japan suffered from OHCA during 20052013 , of which approximately $55 \%$ of the patients had cardiac disease, and approximately $4 \%$ of the patients had experienced a witnessed ventricular fibrillation (VF). ${ }^{1}$ Among those patients with witnessed VF, the proportion of patients with return of spontaneous circulation (ROSC) was $31.4 \%$, and the proportion of patients with survival with favorable

\section{Article p 1842}

neurological outcome, defined as a Cerebral Performance Category (CPC) 1 (good performance) or 2 (moderate disability) on a 5-category scale, ${ }^{2}$ was $20.3 \%$, for which increasing application of public-access defibrillators in Japan has contributed to the improvement in the chance of ROSC. ${ }^{1}$

Based on the efforts of resuscitation by basic life support in the pre- and in-hospital settings, patients with ROSC are

\section{A}

Out-of-Hospital Cardiac Arrest

Collapse-to-ROSC time $\geq 18 \mathrm{~min}$

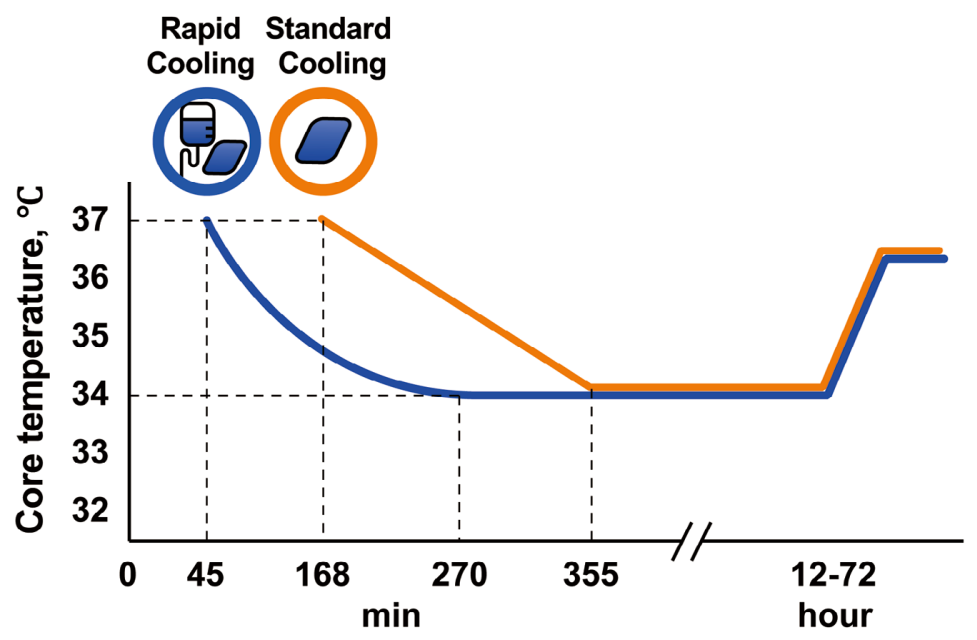

Time from Collapse
B

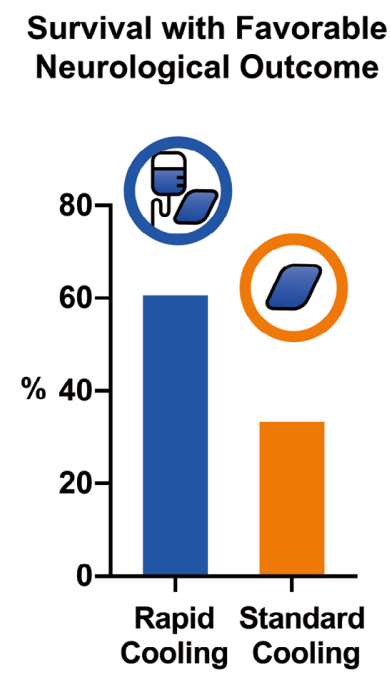

Figure. Central findings from the J-PULSE-HYPO study. (A) Rapid cooling with ice-cold saline infusion followed by standard cooling (e.g., surface cooling or direct blood cooling) was associated with shorter time from collapse to the initiation of cooling and to the attainment of $34^{\circ} \mathrm{C}$ compared with standard cooling alone among patients with out-of-hospital cardiac arrest with collapse-to-return of spontaneous circulation (ROSC) time $\geq 18 \mathrm{~min}$. (B) Rapid cooling with ice-cold saline infusion followed by standard cooling was associated with larger probability of favorable neurological outcome (Cerebral Performance Category $\leq 2$ ) compared with standard cooling alone.

The opinions expressed in this article are not necessarily those of the editors or of the Japanese Circulation Society.

Received July 19, 2021; accepted July 19, 2021; J-STAGE Advance Publication released online August 18, 2021

Department of Cardiovascular Medicine, Kyushu University, Fukuoka, Japan

Mailing address: Tetsuya Matoba, MD, PhD, FJCS, Department of Cardiovascular Medicine, Kyushu University, 3-1-1 Maidashi,

Higashi-ku, Fukuoka 812-8582, Japan. E-mail: matoba@cardiol.med.kyushu-u.ac.jp

All rights are reserved to the Japanese Circulation Society. For permissions, please e-mail: cj@j-circ.or.jp

ISSN-1346-9843 
further treated by advanced life support (ALS). Among patients with cardiovascular shock admitted to a cardiovascular center in Japan, OHCA before hospital arrival is a strong predictor of poor neurological outcome. ${ }^{3-5}$ This situation drives research attempts to improve post-resuscitation care. The ILCOR (International Liaison Committee On Resuscitation) and its member associations, including the Japan Resuscitation Council (JRC), have published the results of systematic reviews and guidelines on ALS for patients' resuscitation from cardiac arrest, ${ }^{6-8}$ including post-resuscitation care that comprises a multidisciplinary approach: (1) targeted temperature management (TTM), (2) optimization of hemodynamics and ventilation, (3) percutaneous coronary intervention, and (4) neurological assessment and care.

The ILCOR Consensus on Science and Treatment Recommendation published in 2015 and 2020 recommends TTM between $32^{\circ} \mathrm{C}$ and $36^{\circ} \mathrm{C}$ as opposed to no TTM for adults with OHCA with or without an initial shockable rhythm who remain unresponsive after ROSC; however, the level of evidence is low. ${ }^{6,7}$ The JRC guideline 2020 identifies several clinical questions regarding an optimal TTM protocol that remain to be answered: (1) optimal target core temperature, (2) optimal duration, (3) optimal timing of TTM induction, (4) clinical benefit of prehospital induction of TTM, and (5) optimal cooling technique. ${ }^{8}$ Because the evidence from randomized controlled trials (RCT) is limited, findings from observational studies are of importance in this area.

In this issue of the Journal, Matsuzaki et $\mathrm{al}^{9}$ examine the association of neurological outcome with the induction of hypothermia by an infusion of cold saline among OHCA patients registered in the J-PULSE-HYPO study registry. The J-PULSE-HYPO study ${ }^{10,11}$ was a multicenter voluntary registry of OHCA patients with post-resuscitation neurological dysfunction in Japan. Matsuzaki et al stratified OHCA patients with collapse-to-ROSC time, a significant factor of prognosis, ${ }^{11}$ and found that among the patients with collapseto-ROSC time $\geq 18 \mathrm{~min}$, rapid cooling with ice-cold saline infusion followed by standard cooling (e.g., surface cooling or direct blood cooling) was associated with shorter time from collapse to the initiation of cooling and to the attainment of $34^{\circ} \mathrm{C}$ compared with standard cooling alone, and importantly, with a higher probability of 30-day favorable neurological outcome (Figure), in contrast to the patients with collapse-to-ROSC time $<18 \mathrm{~min}$ in whom rapid cooling was not associated with neurological outcome.

Very recently, awaited results of the TTM2 trial, an RCT that compared targeted hypothermia at $33^{\circ} \mathrm{C}$ with targeted normothermia among 1,900 patients in a coma after OHCA, has been published, in which targeted hypothermia did not show differences with respect to death and neurological function. ${ }^{2}$ In the TTM2 trial, median time from collapse to ROSC was $25 \mathrm{~min}$, and the median time from collapse to randomization that preceded the initiation of hypothermia was $135 \mathrm{~min}$. Although the TTM2 trial has been assumed to significantly affect the body of evidence regarding the target temperature during TTM, ${ }^{7,8}$ there remain several questions to be addressed. The current observational study by Matsuzaki et al may provide clinical hypotheses that there is a therapeutic window for TTM depending on the time from collapse to ROSC, and that earlier initiation of hypothermia may have benefit regarding neurological outcomes (Figure), which should be clarified by larger, pro- spective and interventional studies.

In conclusion, post-resuscitation care is receiving increasing attention in cardiovascular medicine to obtain better likelihood of survival with good neurological outcome in patients with OHCA. Although current guidelines recommend TTM, pursuit of the optimal protocol of TTM still continues through trials to answer several clinical questions.

\section{Disclosures}

H.T. is an Editorial Member of Circulation Journal.

\section{Conflict of Interest Disclosures}

The authors have no conflicts of interest to disclose related to this editorial comment.

\section{IRB Information / Reference Number}

None.

\section{References}

1. Kitamura T, Kiyohara K, Sakai T, Matsuyama T, Hatakeyama T, Shimamoto T, et al. Public-access defibrillation and out-of-hospital cardiac arrest in Japan. $N$ Engl J Med 2016; 375: 1649-1659.

2. Cummins RO, Chamberlain DA, Abramson NS, Allen M, Baskett PJ, Becker L, et al. Recommended guidelines for uniform reporting of data from out-of-hospital cardiac arrest: The Utstein Style. A statement for health professionals from a task force of the American Heart Association, the European Resuscitation Council, the Heart and Stroke. Circulation 1991; 84: 960-975.

3. Ueki Y, Mohri M, Matoba T, Tsujita Y, Yamasaki M, Tachibana E, et al. Characteristics and predictors of mortality in patients with cardiovascular shock in Japan: Results from the Japanese Circulation Society Cardiovascular Shock Registry. Circ J 2016; 80: 852-859.

4. Sakamoto K, Matoba T, Mohri M, Ueki Y, Tsujita Y, Yamasaki $\mathrm{M}$, et al. Clinical characteristics and prognostic factors in acute coronary syndrome patients complicated with cardiogenic shock in Japan: Analysis from the Japanese Circulation Society Cardiovascular Shock Registry. Heart Vessels 2019; 34: 1241 - 1249.

5. Matoba T, Sakamoto K, Nakai M, Ichimura K, Mohri M, Tsujita $\mathrm{Y}$, et al. Institutional characteristics and prognosis of acute myocardial infarction with cardiogenic shock in Japan: Analysis from the JROAD/JROAD-DPC database. Circ J 2021; 85: $1797-$ 1805 .

6. Callaway CW, Soar J, Aibiki M, Böttiger BW, Brooks SC, Deakin CD, et al. Part 4: Advanced life support: 2015 International consensus on cardiopulmonary resuscitation and emergency cardiovascular care science with treatment recommendations. Circulation 2015; 132: S84-S145.

7. Berg KM, Soar J, Andersen LW, Böttiger BW, Cacciola S, Callaway CW, et al. Adult Advanced Life Support: 2020 International Consensus on Cardiopulmonary Resuscitation and Emergency Cardiovascular Care Science With Treatment Recommendations. Circulation 2020; 142: S92-S139.

8. Akasaka S, Atsumi I, Katoh M, Saito N, Takahashi K, Takahashi $\mathrm{H}$, et al. Adult Advanced Life Support: JRC Guidelines for Resuscitation 2020 [in Japanese] Tokyo: Igaku Shoin, 2021; 48-150.

9. Matsuzaki M, Matsumoto N, Nagao K, Sawano H, Yokoyama $\mathrm{H}$, Tahara $\mathrm{Y}$, et al. Impact of induced therapeutic hypothermia by intravenous infusion of ice-cold fluids after hospital arrival in comatose survivors of out-of-hospital cardiac arrest with initial shockable rhythm. Circ J 2021; 85: 1842-1848

10. Yokoyama H, Nagao K, Hase M, Tahara Y, Hazui H, Arimoto $\mathrm{H}$, et al. Impact of therapeutic hypothermia in the treatment of patients with out-of-hospital cardiac arrest from the J-PULSEHYPO study registry. Circ J 2011; 75: 1063-1070.

11. Soga T, Nagao K, Sawano H, Yokoyama H, Tahara Y, Hase M, et al. Neurological benefit of therapeutic hypothermia following return of spontaneous circulation for out-of-hospital non-shockable cardiac arrest. Circ J 2012; 76: 2579-2585.

12. Dankiewicz J, Cronberg T, Lilja G, Jakobsen JC, Levin H, Ullén $\mathrm{S}$, et al. Hypothermia versus normothermia after out-of-hospital cardiac arrest. $N$ Engl J Med 2021; 384: 2283-2294. 